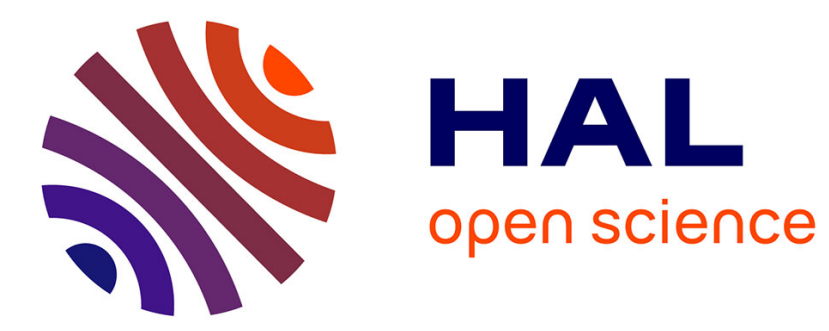

\title{
Techniques pour la détermination en traction des conditions d'adoucissement du fer par alliage
}

\author{
J.P. Peyrade, P. Chomel, J.P. Cottu, P. Astie, Y. Philippe
}

\section{To cite this version:}

J.P. Peyrade, P. Chomel, J.P. Cottu, P. Astie, Y. Philippe. Techniques pour la détermination en traction des conditions d'adoucissement du fer par alliage. Revue de Physique Appliquée, 1977, 12 (6), pp.937-947. 10.1051/rphysap:01977001206093700 . jpa-00244264

\section{HAL Id: jpa-00244264 https://hal.science/jpa-00244264}

Submitted on 1 Jan 1977

HAL is a multi-disciplinary open access archive for the deposit and dissemination of scientific research documents, whether they are published or not. The documents may come from teaching and research institutions in France or abroad, or from public or private research centers.
L'archive ouverte pluridisciplinaire HAL, est destinée au dépôt et à la diffusion de documents scientifiques de niveau recherche, publiés ou non, émanant des établissements d'enseignement et de recherche français ou étrangers, des laboratoires publics ou privés. 


\author{
Classification \\ Physics Abstracts \\ $0.605-9.116-9.170$
}

\title{
TECHNIQUES POUR LA DÉTERMINATION EN TRACTION DES CONDITIONS D'ADOUCISSEMENT DU FER PAR ALLIAGE
}

\author{
J. P. PEYRADE, P. CHOMEL, J. P. COTTU, P. ASTIE et Y. PHILIPPE
}

Laboratoire de Physique des Solides (*), I. N. S. A., Département de Physique avenue de Rangueil, 31077 Toulouse-Cedex, France

(Reçu le 25 novembre 1976, révisé le 24 janvier 1977, accepté le 23 février 1977)

\begin{abstract}
Résumé. - Cet article décrit les techniques que nous avons mises au point en vue de l'étude, par traction à basse température, du phénomène d'adoucissement du fer par alliage. Elles permettent, en particulier, d'élaborer du fer et des alliages de haute pureté, de préparer des échantillons polycristallins à grains fins, et de déterminer avec précision les contraintes d'écoulement plastique et les paramètres d'activation.
\end{abstract}

Abstract. - Several experimental methods have been developped in order to study the alloy softening effect in iron through tensile tests at low temperatures. These methods are the following : elaboration of very pure iron and alloys, fine grain sample preparation, and accurate determination of flow stresses and activation parameters.

L'étude des phénomènes d'adoucissement des métaux de structure cubique centrée, à basse température, soulève de nombreuses controverses. D'une part, les résultats expérimentaux peuvent être divergents, d'autre part, les interprétations reposent sur des points de vue différents [1], [2], le problème étant de savoir si l'adoucissement a une origine intrinsèque (action directe des atomes d'alliage sur la mobilité des dislocations) ou une origine extrinsèque (interactions entre atomes d'impuretés et d'éléments d'alliage).

Les premières expériences que nous avons effectuées pour tenter de répondre à cette question $[3,4]$ nous ont amenés à conclure que les réponses apportées dépendaient très fortement de la manière dont l'étude expérimentale était menée.

Le but de cet article est donc de décrire aussi complètement que possible les techniques que nous avons mises au point et qui nous paraissent nécessaires à l'étude de ces phénomènes d'adoucissement. Elles comprennent notamment :

- L'élaboration de fer et d'alliages de fer de haute pureté ;

- L'obtention, à défaut de monocristaux, d'échantillons polycristallins dont la taille de grain soit fine et identique d'un alliage à l'autre ;

- La détermination des contraintes d'écoulement plastique et des paramètres d'activation de la déformation plastique à basse température avec une grande précision.

(*) Associé au C. N. R. S.
Nous estimons, par conséquent, que les essais de traction et de relaxation doivent être effectués à l'aide d'un montage qui n'introduise pas d'effets de relaxation parasites, d'origine mécanique ou thermique, et qu'il est nécessaire de s'assurer de la reproductibilité des résultats obtenus à partir de plusieurs éprouvettes.

Nous limiterons l'exposé des résultats au cas du fer pur, le cas des alliages devant faire l'objet d'autres publications.

1. Elaboration du matériau. - 1.1 TECHNIQUE DE PURIFICATION DU FER. - Les méthodes de production du fer de haute pureté sont toutes fondées sur l'emploi de processus de purification successifs, encore appelés processus de purification en cascade [5].

Le choix d'un fer de départ industriellement pur, est suivi d'une purification par voie chimique se terminant le plus souvent par un processus d'échange d'ions [6], [7], [8], [9]. On prépare ensuite un fer électrolytique [10], [11], [12], [13] auquel on fait subir une purification ultime par zone fondue [10], [14], [15], [16], [17] ou au chalumeau à plasma [18], [19].

Le fer que nous utilisons est produit en grande quantité par le laboratoire de métallurgie de l'Ecole des Mines de Saint-Etienne. D'excellente pureté, car ayant subi un cycle de purification sur résines échangeuses d'ions [6], il se présente sous forme d'une éponge de fer, incomplètement réduite. Il contient donc beaucoup d'oxygène, des impuretés métalliques en concentration inférieure à $1 \mathrm{ppm}$, des impuretés métalloïdiques en concentration inférieure à $2 \mathrm{ppm}$ (sauf $\mathrm{C}, \mathrm{O}, \mathrm{S}$ ), du silicium en concentration inférieure 
à $20 \mathrm{ppm}$ ( $<10 \mathrm{ppm}$ depuis peu). Cette éponge doit être refondue, et si possible purifiée.

L'examen des méthodes classiques de refusion du fer [23] et des atmosphères habituelles de fusion, nous a conduits à utiliser une technique de fusion par haute fréquence, sur nacelle de cuivre refroidie, et sous atmosphère soigneusement adaptée.

Nous avons écarté la technique de fusion en creuset réfractaire, à cause des risques de contamination par le creuset lui-même, ainsi que les méthodes de fusion au chalumeau à plasma [19] ou en lévitation pure [20], [21], [22], [23], celles-ci nous limitant à la production de lingots d'une dizaine de grammes.

Sachant que certains éléments en solution dans le fer sont difficiles à éliminer par la méthode de la zone fondue (leur coefficient de ségrégation à l'équilibre, $k_{0}$, est compris entre 0,5 et 1 ; par exemple $\mathrm{Al}, \mathrm{Co}$, $\mathrm{Cr}, \mathrm{Cu}, \mathrm{Mn}, \mathrm{Mo}, \mathrm{Si}, \mathrm{Sn}, \mathrm{V}, \mathrm{W}, \mathrm{Zn}$ [24], [25]) nous avons dû envisager une autre méthode, plus efficace, pour éliminer la principale impureté de notre matériau, à savoir, le silicium.

En nous appuyant sur le fait que les éléments les plus facilement oxydables, sous pression partielle d'oxygène, sont, par ordre décroissant : $\mathrm{Ca}, \mathrm{Mg}, \mathrm{Li}$, $\mathrm{Al}, \mathrm{Ti}, \mathrm{Si}, \mathrm{V}, \mathrm{Mn}, \mathrm{Cr}, \mathrm{Na}, \mathrm{Zn}, \mathrm{K}, \mathrm{P}$ [26] et sur les travaux de B. F. Oliver et E. W. Troy [27] nous avons pensé à éliminer le silicium en réalisant un superraffinage par oxydation.

1.1.1 Superraffinage par oxydation. - Pour éliminer le silicium, très réactif, d'un métal peu réactif avec l'oxygène comme le fer, on soumet ce dernier à une oxydation contrôlée, à température assez élevée pour augmenter la cinétique du phénomène.

a) Bases thermodynamiques de la méthode. - Puisque le fer de départ est déjà très pur, nous admettrons que la scorie est formée en grande partie du $\mathrm{SiO}_{2}$ solide et du $\mathrm{FeO}$ liquide à la température de l'oxydation (non miscible dans le fer).

L'éponge de fer contenant beaucoup d'oxygène, la solution liquide fer-oxygène-silicium est saturée en oxygène. Le silicium en solution se combine à l'oxygène dissous, à $1535^{\circ} \mathrm{C}<T<1610^{\circ} \mathrm{C}$ selon la réaction :

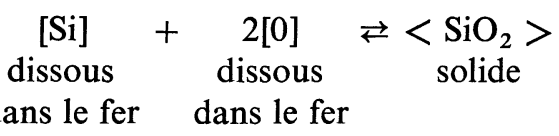

Cet équilibre, fonction de la température est caractérisé :

- par la variation d'énergie libre standard [25]

$$
\Delta G_{0}=-139600+67,25 T-4,15 T \log T
$$

- et par la constante d'équilibre :

$$
K_{T}=\frac{\left.a<\mathrm{SiO}_{2}\right\rangle}{a \mathrm{Si}_{\mathrm{Fe}} \cdot a^{2}[\mathrm{O}]_{\mathrm{Fe}}} .
$$

Sachant qu'à l'équilibre, $\Delta G_{0}=-R T \log K_{T}$, nous pouvons calculer $K_{T}$. Connaissant, à la température $T$ :

- les paramètres d'interaction Si-O en présence de fer [29],

- la limite de solubilité de l'oxygène dans le fer, $C_{0}$, nous pouvons en déduire les coefficients d'activité $\mathrm{du}$ silicium, $f_{\mathrm{Si}}$, et de l'oxygène, $f_{\mathrm{O}}$, puis la concentration limite en silicium :

$$
\underset{\% \text { en poids }}{C_{\mathrm{Si}}}=\frac{1}{K_{T} \cdot f_{\mathrm{Si}} \cdot f_{\mathrm{o}}^{2} \cdot C_{\mathrm{O}}^{2}}
$$

soit $1,9 \mathrm{ppm}$ en poids à $1600^{\circ} \mathrm{C}$, et $0,47 \mathrm{ppm}$ en poids à $1535^{\circ} \mathrm{C}$, pour une solution liquide saturée en oxygène $(0,23 \%$ en poids).

b) Réalisation de l'oxydation - L'éponge de fer (200 g environ) est d'abord refondue au four H. F., sur une nacelle de cuivre refroidie à l'eau, et sous atmosphère d'hélium purifié. Une zone liquide de $2 \mathrm{~cm}$ de large est déplacée alternativement d'un bout à l'autre du lingot, à la vitesse de $24 \mathrm{~cm} / \mathrm{H}$. Le lingot est retourné entre chaque passe ainsi effectuée à une vitesse très supérieure à celles qui sont utilisées dans les méthodes de purification par zone fondue et il n'y a pratiquement pas de ségrégation des impuretés lors de ces opérations. L'oxygène contenu dans l'éponge réagit alors avec le fer et avec les impuretés en donnant des scories superficielles.

c) Extraction des scories. - Le lingot (Fig. 1) est débarrassé de la couche de scories par décapage mécanique à la brosse, puis attaque chimique dans l'acide chlorhydrique pur. Les opérations fusiondécapage sont effectuées trois fois afin d'atteindre l'état d'équilibre thermodynamique scorie-solution liquide.

d) Analyse des scories. - L'étude effectuée au microanalyseur ionique du laboratoire nous a permis de déterminer la nature des éléments présents dans les scories.

Les spectres d'émission obtenus par bombardement du fer pur et des scories formées à sa surface, à l'aide d'ions primaires $\mathrm{A}^{+}$sont reproduits sur la figure 2 .

Nous remarquons, dans le fer pur, l'existence de $\mathrm{Na}, \mathrm{O}_{2}, \mathrm{Al}, \mathrm{K}$ et $\mathrm{Ca}$ à l'état de traces. Le spectre des scories, tracé avec la même sensibilité, sur une plage voisine de l'échantillon, révèle l'accumulation en surface des oxydes de $\mathrm{Mg}, \mathrm{Al}, \mathrm{Si}, \mathrm{K}, \mathrm{Ca}, \mathrm{Ti}, \mathrm{Cr}, \mathrm{V}$, $\mathrm{Mn}, \mathrm{Ni}, \mathrm{Co}, \mathrm{Zn}, \mathrm{Ge}, \mathrm{Mo}, \mathrm{Na}$ et la présence d'oxyde de fer. Ce sont les oxydes des éléments facilement oxydables, déjà cités.

Le taux d'émission variant selon l'élément considéré, nous ne pouvons pas comparer entre eux les pics d'abscisse différente. Par contre, la comparaison des pics de même abscisse est possible et indique la présence d'une quantité importante de silicium dans 


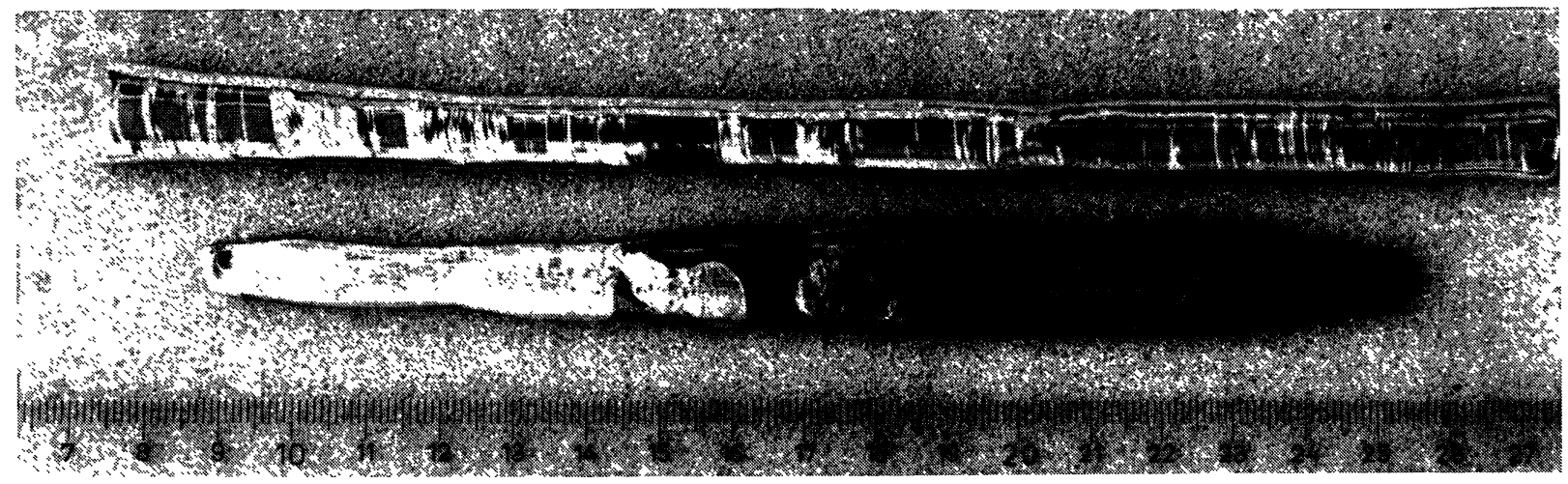

Fig. 1. - Lingot de fer. Etat oxydé et état final.

[Iron ingot. Oxydised and final states.]

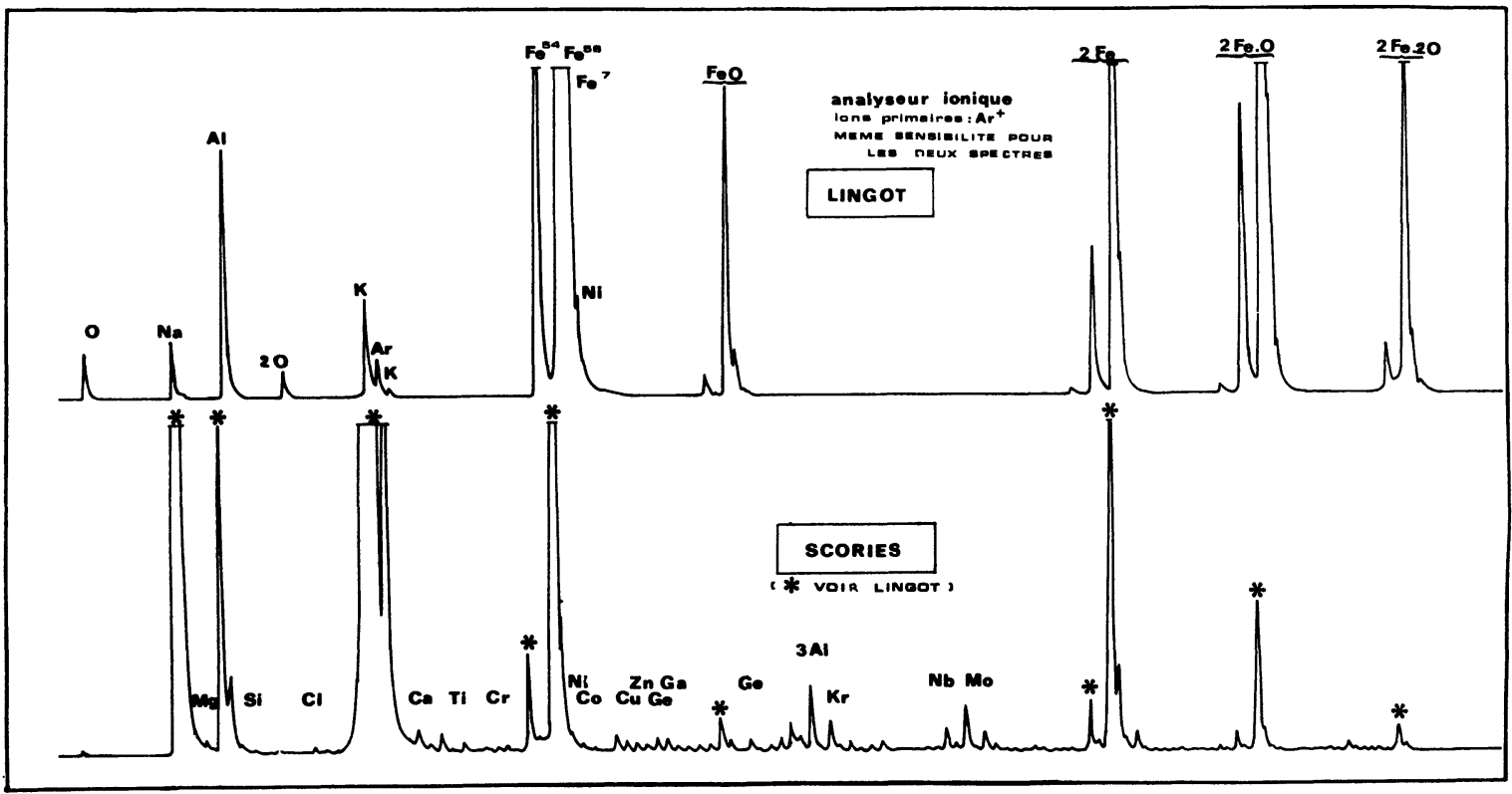

Fig. 2. - Spectres d'émission du fer et des scories.

[Emission spectrum of iron and of slag.]

les scories, ce qui confirme la purification de notre matériau.

De nombreuses inclusions d'oxyde de fer, $\mathrm{FeO}$, s'étant formées lors de la solidification, une opération de réduction du fer est nécessaire à l'issue des traitements précédents.

1.1.2 Réduction du fer. - L'hydrogène, facilement purifiable, est un excellent réducteur à une température supérieure à $825^{\circ} \mathrm{C}$. Le lingot est donc refondu au four $\mathrm{HF}$, sur nacelle de cuivre refroidie, sous atmosphère d'hydrogène purifié, et à la même vitesse que précédemment. Le lingot est retourné entre chaque passe.

1.1.3 Contrôle de la pureté par mesure de résistivité. - Le rapport de résistivité $R=\rho 4,2 \mathrm{~K} / \rho 298 \mathrm{~K}$ permet d'avoir une idée de la pureté globale du fer [31].
Certains éléments ont peu d'influence sur ce rapport, comme l'oxygène, d'autres beaucoup plus comme le carbone.

Nous avons obtenu, sous champ magnétique nul, un rapport $R=100 \times 10^{-4}$ sur un fer pur (recristallisé $10 \mathrm{~h}$ à $750^{\circ} \mathrm{C}$ sous $\mathrm{H}_{2}$ ), obtenu sans superraffinage préalable et de $42 \times 10^{-4}\left({ }^{1}\right)$ après superraffinage et réduction. Nous constatons donc que l'élimination des impuretés se traduit bien par une diminution du rapport de résistivité. Nous pensons toutefois que la valeur absolue de celui-ci ne constitue pas une mesure suffisante de la pureté en raison de

(1) Cette valeur, mesurée sous champ magnétique nul tombe à $23,5 \times 10^{-4}$ pour $H=1000 \mathrm{Oe}$. Nous remercions M. O. Dimitrov, Directeur de Recherche au Laboratoire du C. N. R. S. de Vitry, d'avoir bien voulu effectuer cette mesure à notre intention. 
l'influence de nombreux autres paramètres sur sa valeur (composition initiale de l'éponge, écrouissage, taille de grain, ...).

1.1.4 Analyse chimique $d u$ fer. - Une analyse par activation a confirmé la très faible teneur de notre fer en éléments d'insertion [32] :

$$
\begin{aligned}
& \mathrm{C}=0,3 \mathrm{ppm} \text { en poids } \\
& \mathrm{N}=0,34 \mathrm{ppm} \text { en poids } \\
& \mathrm{O}=0,52 \mathrm{ppm} \text { en poids. }
\end{aligned}
$$

L'analyse des autres éléments par spectrométrie de masse à étincelle conduit aux résultats reportés

\begin{tabular}{|c|c|c|}
\hline Eléments & $\underset{(*)}{\text { Analyse }}$ du fer & $\begin{array}{l}\text { Analyse de l'éponge } \\
(* * *)\end{array}$ \\
\hline $\mathrm{C}$ & $0,3(* *)$ & - \\
\hline $\mathbf{N}$ & $0,3(* *)$ & - \\
\hline $\mathrm{O}$ & $0,5(* *)$ & 2000 \\
\hline $\mathbf{P}$ & 0,2 & $<2$ \\
\hline S & $\leqslant 5$ & $<2$ \\
\hline $\mathrm{Cl}$ & 1 & - \\
\hline $\mathbf{K}$ & 2 & - \\
\hline $\mathrm{Ca}$ & 2 & - \\
\hline $\mathrm{Na}$ & 4 & - \\
\hline $\mathrm{Mg}$ & $\leqslant 2$ & - \\
\hline $\mathrm{Si}$ & 5 & $<20$ \\
\hline $\mathrm{Ti}$ & 5 à 10 & - \\
\hline V & 0,1 & - \\
\hline $\mathrm{Cr}$ & 1 & $<1$ \\
\hline Mn & 1 & $<1$ \\
\hline $\mathrm{Ni}$ & $\leqslant 0,5$ & $<1$ \\
\hline Co & $\leqslant 0,1$ & $<1$ \\
\hline $\mathrm{Cu}$ & $\leqslant 0,2$ & 1,4 \\
\hline $\mathrm{Ga}$ & $\leqslant 0,2$ & 2,7 \\
\hline As & $\leqslant 0,1$ & $<1$ \\
\hline $\mathrm{Zr}$ & $\leqslant 0,1$ & $<1$ \\
\hline $\mathrm{Nb}$ & $\leqslant 0,4$ & - \\
\hline $\mathrm{Ag}$ & $\leqslant 0,3$ & $<1$ \\
\hline $\mathrm{Pt}$ & $\leqslant 0,3$ & - \\
\hline $\mathrm{Pb}$ & 0,4 à 1 & - \\
\hline $\mathrm{Bi}$ & 0,01 & - \\
\hline $\mathrm{Sb}$ & non dosé & $<1$ \\
\hline $\mathrm{Au}$ & non dosé & $<1$ \\
\hline Mo & non dosé & $<1$ \\
\hline $\mathrm{Al}$ & non dosé & $<1$ \\
\hline W & non dosé & $<1$ \\
\hline $\mathrm{Zn}$ & non dosé & $<1$ \\
\hline $\mathrm{Se}$ & non dosé & $<1$ \\
\hline $\mathrm{Hg}$ & non dosé & $<1$ \\
\hline
\end{tabular}
tableau I.

\section{TABLEAU I}

(*) Par spectrographie de masse (ppm en poids). $(* *)$ Par activation.

$(* * *)$ Teneur de l'éponge de départ donnée par le fournisseur (ppm en poids).

L'examen des résultats précédents et l'étude des données disponibles dans la littérature (cf. [39]) nous permet de penser que nous disposons d'un matériau de pureté suffisante pour aborder l'étude de l'adoucissement du fer par alliage dans de bonnes conditions. La purification ultérieure de notre métal par zone fondue n'est donc pas envisagée pour le moment.

1.2 Préparation des alliages. - Deux techniques de préparation d'alliage ont été mises au point. Elles ont été décrites en détail par ailleurs [33], [34], [35].

- L'une nous permet de préparer, en phase liquide, des alliages fer-élément de substitution $X$, par lévitation en concentrateur de champ, sous atmosphère d'hydrogène ou d'hélium de haute pureté [33]

- L'autre nous permet d'introduire un troisième élément en phase solide, le carbone. Nous maintenons pour cela l'échantillon, à température donnée, dans une atmosphère $\mathrm{CH}_{4}-\mathrm{H}_{2}$.

L'étude thermodynamique des équilibres gaz-solide, $\mathrm{CH}_{4}+\mathrm{H}_{2} \rightleftarrows \mathrm{Fe}+\mathrm{X}+\mathrm{C}$, permet de montrer que l'on peut maîtriser la teneur en carbone des échantillons traités depuis le ppm jusqu'à la limite de solubilité du carbone en phase $\alpha$ ou $\gamma$, à la température du traitement, en réglant la teneur en $\mathrm{CH}_{4}$ de la phase gazeuse [34], [35].

Ainsi, grâce aux techniques que nous venons de décrire, ou de rappeler, nous disposons :

- d'un matériau de référence de très haute pureté, exempt d'interstitiels ;

— d'alliages Fer-X, non piégés à teneur en interstitiels très faible ;

- d'alliages Fer-C et Fer-X-C à teneur en interstitiels variable.

2. Préparation des éprouvettes. Recristallisation. Le lingot purifié se présente sous forme de barreau de 100 à $160 \mathrm{~g}$, de section elliptique légèrement aplatie à la partie inférieure $(8 \mathrm{~mm} \times 10 \mathrm{~mm}$ environ), de 15 à $20 \mathrm{~cm}$ de long. Sa structure est constituée de gros grains allongés selon l'axe du barreau, de 1 à $8 \mathrm{~mm}$ de long avec une section transversale qui peut atteindre $2 \mathrm{~mm}^{2}$. Nous devons alors le transformer en éprouvettes de traction à grains fins (Fig. 3).

2.1 FORME DES ÉPROUVETTES. - Nous avons choisi de préparer des éprouvettes plates de $0,5 \mathrm{~mm}$ d'épaisseur, de $1 \mathrm{~mm}^{2}$ de section dans la partie utile, en nous guidant sur les considérations suivantes :

- Eviter au maximum la pollution au cours de la préparation : le laminage de plats peut se faire beaucoup plus proprement que celui de fils au laminoir à gorges ;

- Conserver une section suffisante par rapport à la taille de grain espérée et obtenue ;

- Obtenir, par laminage, un écrouissage important et uniforme sur toute l'épaisseur de l'échantillon afin d'assurer l'homogénéité de la taille du grain final, après recristallisation ; 


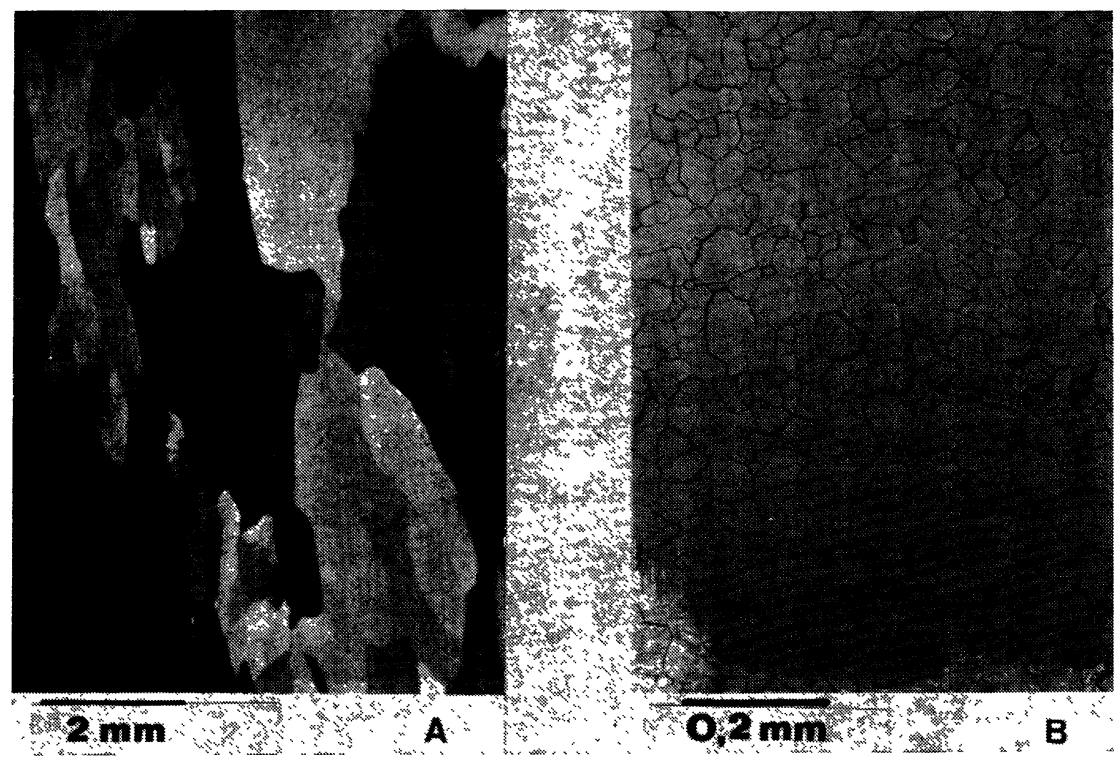

FIG. 3. - Micrographies du fer ; A : lingot brut, B : éprouvette recristallisée.

[Iron micrographs ; A. Initial ingot ; B. Final sample.]

- Utiliser les mêmes éprouvettes pour l'étude du phénomène d'adoucissement par traction et par frottement intérieur ;

- Economiser le matériau.

Les dimensions des têtes et des congés de raccordement ont été déterminées de telle façon que la déformation reste bien localisée dans la partie utile. Nous avons vérifié en particulier que l'allongement de celle-ci, $L_{0}=12 \mathrm{~mm}$, et le déplacement relatif des mors étaient identiques dans la gamme de déformation utilisée.

Un polissage électrolytique soigné, avant traction, permet de réduire la tendance au mâclage particulièrement favorisée par cette forme d'éprouvette.

2.2 Affinage du grain. - Notre méthode repose sur l'application successive de traitements d'écrouissage et de recristallisation. Les premières tentatives que nous avons effectuées ont montré que :

- L'affinage du grain dépend beaucoup de la pureté, ce qui rend difficile la transposition des résultats fournis par d'autres auteurs [36], [37].

- Le laminage, habituellement utilisé comme méthode d'écrouissage, ne permet pas d'atteindre des taux de déformation suffisants pour affiner le grain d'un métal très pur. Les anciens gros grains ont une forte tendance à redonner des gros grains. C'est pourquoi nous avons adopté la technique suivante :

- Compression, à la presse, du barreau initial. Six réductions relatives de l'épaisseur, de $20 \%$ environ, sont effectuées, alternativement, dans deux directions perpendiculaires. La section finale est approximativement carrée, de $8 \mathrm{~mm}$ de côté ;

- Un traitement de recristallisation de $1 \mathrm{~h}$ à $600^{\circ} \mathrm{C}$ REVUE DE PHYSIQUe APPLIQUÉE. - T. 12, N 6, JUIN 1977 sous hydrogène purifié conduit à une taille de grain de 200 à $400 \mu \mathrm{m}$;

- Une nouvelle série de compressions conduit à une section de $4 \times 20 \mathrm{~mm}^{2}$. Un laminage amène alors l'échantillon à l'épaisseur $0,5 \mathrm{~mm}$;

- La recristallisation finale, de $3 \mathrm{~h}$ à $530^{\circ} \mathrm{C}$ sous hydrogène purifié se traduit par une taille de grain homogène de $50 \mu \mathrm{m}$ environ (Fig. 3).

Tous les traitements thermiques sont précédés d'un nettoyage énergique et d'un polissage électrolytique des échantillons. Cette technique est également applicable dans le cas des alliages sous réserve d'effectuer quelques aménagements.

3. Dispositifs de traction à basse température $\left({ }^{2}\right)$. -

3.1 Le mONTAGE méCANIQUe. - Nos objectifs, lors de cette réalisation, étaient les suivants :

- Eviter les effets de relaxation parasites d'origine mécanique en conservant la possibilité de réaliser l'alignement du montage lors de la mise en place de l'échantillon ;

- Pouvoir effectuer des variations rapides de température sans consommation excessive de fluide cryogénique ;

- Obtenir une bonne stabilité thermique du montage et de l'échantillon pendant un essai.

(2) Le montage de traction et le cryostat à azote liquide ont été conçus et mis au point en collaboration avec N. S. Askénazy, Responsable du Service Interlaboratoires des champs magnétiques intenses de Toulouse, à l'occasion d'une étude des caractéristiques mécaniques de matériaux composites isolants, à basse température [38]. 


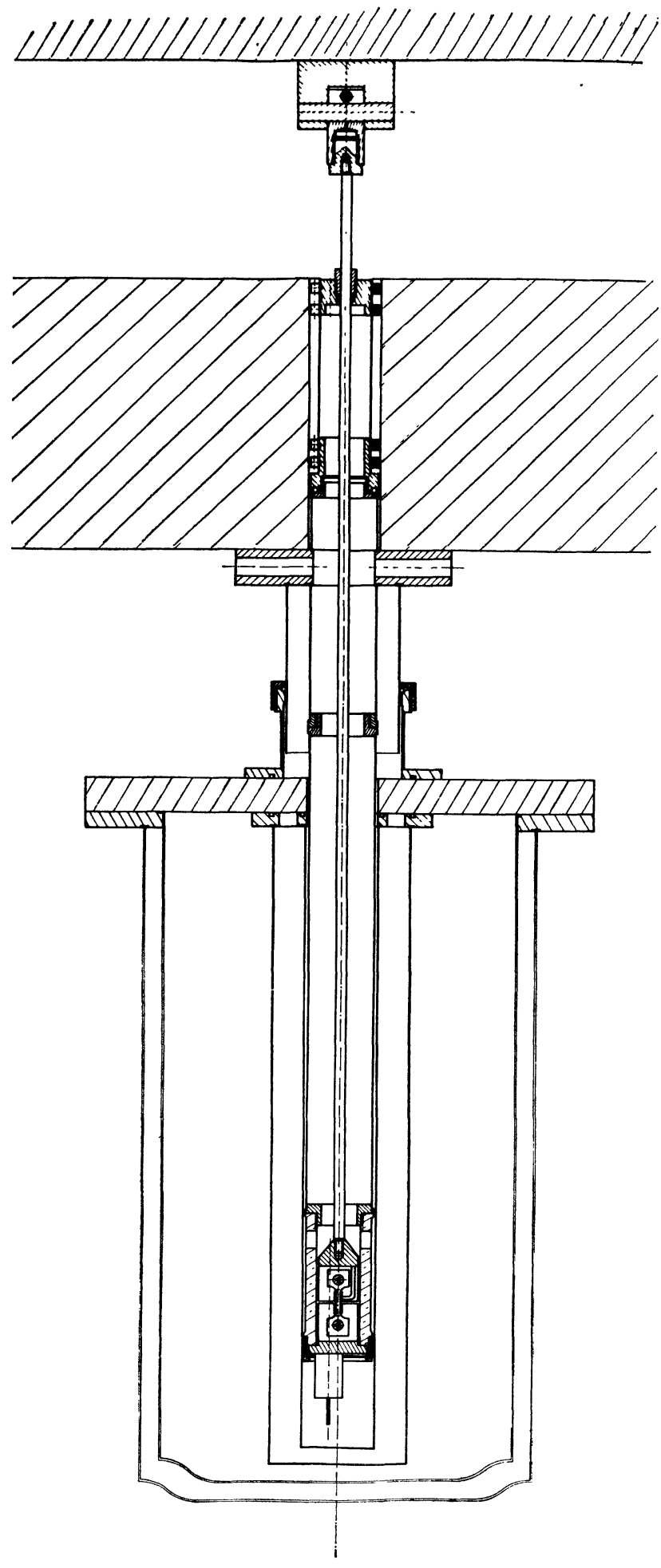

Fig. 4. - Montage de traction et cryostat.

[Cryostat and tensile test device.]

Le montage retenu est représenté sur la figure 4. Il est placé sur une machine de traction Instron TTDM équipée d'une cellule de charge de $500 \mathrm{daN}$.

- Les rotules et les articulations libres habituellement placées sur les lignes d'amarrage sont à l'orjgine d'effets de relaxation parasites [39] qui rendent imprécise la détermination du volume d'activation par relaxation mécanique [40]. L'alignement du montage est donc réalisé à l'aide des articulations placées au niveau de la cellule de charge. Celles-ci sont alors bloquées, ainsi que tous les filetages assurant la liaison des différentes parties de ce dispositif.

- La tige de traction, $\varnothing 6$, est en TA 6 V. Le mors inférieur est appuyé sur un tube fin, $\varnothing$ 36-37 en acier inoxydable NS $22 \mathrm{~S}$.

Nous avons choisi le TA $6 \mathrm{~V}$ en raison de sa mauvaise conductibilité thermique, de sa limite élastique élevée à basse température, et de son faible module d'élasticité. Une très grande souplesse latérale du montage ( $1 \mathrm{~N} / \mathrm{mm}$ au niveau de l'éprouvette) compense très largement l'absence d'articulation libre au niveau de la cellule de charge.

La forme tubulaire de la partie comprimée du montage permet de limiter la section, donc la conduction thermique, sans risque de flambement.

- Les mors sont en cuivre-béryllium et la partie inférieure du tube de compression en cuivre pur, ce qui favorise la stabilité et l'homogénéité de la température de l'éprouvette pendant l'essai.

3.2 Le CR YOSTAT ET LE DiSPOSITIF DE RÉGULATION. Le cryostat représenté sur la figure 4 est destiné aux essais effectués entre $77 \mathrm{~K}$ et $300 \mathrm{~K}$. Il a été réalisé au laboratoire et se compose d'une enceinte à azote liquide, de forme annulaire, et d'un fourreau central à double paroi qui peut être vidé ou rempli d'hélium gazeux selon le degré d'échange thermique désiré. Le montage mécanique peut lui-même être placé sous vide ou sous hélium gazeux, grâce à la présence d'un soufflet d'étanchéité de grande souplesse, à la partie supérieure.

La régulation est obtenue au moyen d'une chaufferette (Fil de manganin $\varnothing 0,2 \mathrm{~mm}$ ) de $40 \Omega$ bobinée sur la pièce de cuivre inférieure et d'un régulateur P. I. D. THOR 3010 II. La sonde de régulation est placée dans l'épaisseur de la pièce en cuivre. Dans ces conditions, il est possible d'obtenir rapidement toute température comprise entre $220 \mathrm{~K}$ et $77 \mathrm{~K}$, d'effectuer, notamment au-dessous de $180 \mathrm{~K}$, un saut de température de $20 \mathrm{~K}$ en $20 \mathrm{~min}$. environ, y compris le temps de stabilisation du montage (dérive inférieure à $0,1 \mathrm{daN} / \mathrm{min}$.). Nous pouvons ainsi effectuer jusqu'à 20 essais de traction et de relaxation en balayant la gamme $300 \mathrm{~K}-77 \mathrm{~K}-300 \mathrm{~K}$ au cours d'une même journée, sur une même éprouvette. L'addition d'azote liquide est alors de 1,5 litre environ, soit la moitié de la capacité du cryostat.

3.3 LeS DISPOSITIFS DE MESURE. - 3.3.1 Mesure de la température. - Une sonde de mesure (résistance de platine) est placée à l'intérieur du mors supérieur, au contact de l'éprouvette. Alimentée en courant constant à $10^{-4}$ près, elle permet de déterminer la température de l'échantillon à 0,1 degré près, précision très suffisante pour l'étude effectuée. 
3.3.2 Mesure de l'allongement. - Un transformateur différentiel est fixé rigidement au mors inférieur. Le noyau du transformateur est relié au mors supérieur par une tige filetée dont la rotation permet le réglage du zéro. Ce dispositif, alimenté par un pont Philips, type PR 9308, fournit une mesure du déplacement relatif des mors à $0,1 \mu \mathrm{m}$ près. Nous avons pu vérifier par ailleurs que ce déplacement correspond bien à l'allongement de la partie utile de nos éprouvettes. Grâce à cet équipement, nous pouvons

- mesurer directement les allongements de traction, et de relaxation,

- vérifier, à chaque essai, que la machine se comporte bien comme un ressort parfait (absence de relaxation parasite),

- déterminer, la rigidité de la machine seule, $d$, qui intervient dans le calcul du volume d'activation [40], [41].

4. Recherche des conditions d'observation de l'adoucissement par traction. - 4.1. Présentation DU PROBLÈME. - L'observation des effets produits par l'addition de quelques dizaines de ppm d'un élément d'alliage interstitiel, ou de quelques pour cent d'un substitutionnel, sur la déformation plastique du fer à basse température, rencontre quelques difficultés.

Le principal problème à résoudre est celui du choix d'une grandeur susceptible de traduire quantitativement cet effet. Ce choix varie notablement d'une étude à l'autre, les différents auteurs représentant l'adoucissement par la diminution de la limite élastique sans définir celle-ci, de la limite élastique inférieure lorsqu'il existe une chute de charge en début de traction, ou de la contrainte d'écoulement plastique (C. E. P.) à $0,2 \%$ ou à $0,5 \%$ de déformation. Dans tous les cas, l'existence d'un stade de microdéformation dont l'étendue varie avec la température de l'essai, la composition et la structure de l'échantillon, semble négligée. En particulier, lorsque la microdéformation du fer pur est de l'ordre de $0,5 \%$ à $2 \%$ à $77 \mathrm{~K}$, selon la structure de l'échantillon, alors qu'elle est beaucoup plus faible dans le cas des alliages, de l'ordre de $0,1 \%$ à $0,5 \%$ [4], le seul choix possible est de comparer les C. E. P. correspondant à une valeur élevée de l'allongement $(\geqslant 1 \%)$ ou mieux encore, l'ensemble de la courbe de traction du métal pur et de l'alliage, à une température donnée.

Une seconde difficulté est liée à la faible intensité de l'effet d'adoucissement produit par un élément d'alliage en solution (de l'ordre de 4 à $5 \times 10^{7} \mathrm{~Pa}$ ). Il est donc nécessaire, en vue d'une étude précise du phénomène physique correspondant, de déterminer d'abord les conditions de température, teneur en élément d'alliage, structure des échantillons, pour lesquelles l'intensité de l'adoucissement est maximale. Pour cela, nous devons aussi disposer d'échantillons de très haute pureté, de structure bien définie, ne différant les uns des autres que par leur composition chimique. Notre description des méthodes de préparation du fer pur et de ses alliages montre qu'il est matériellement difficile d'élaborer une grande quantité d'échantillons, en respectant ces exigences. Nous ne disposerons donc que d'un petit nombre d'éprouvettes, de petites dimensions.

Nous avons alors imaginé un programme d'essais qui permet de déterminer, avec le maximum de rigueur, les conditions dans lesquelles le phénomène d'adoucissement est observé, en n'utilisant que quelques échantillons. Cette méthode repose sur l'utilisation d'un même échantillon à plusieurs températures, pour déterminer les courbes de traction. Elle permet en outre de contrôler par traction l'identité des échantillons, d'éliminer l'éprouvette exceptionnellement mauvaise (taille de grain hétérogène, par exemple) et de réduire la dispersion des résultats associée à l'imprécision des mesures de la section $\left(1 \mathrm{~mm}^{2}\right.$ environ mesurée à 3 ou $4 \%$ près).

4.2 MÉthode De CONTRôle PAR TRACTION DE L'IDENTITÉ DES ÉCHANTILlONS. - 4.2.1 Programme d'essais (Fig. 5). - Chaque éprouvette est prédéfor-

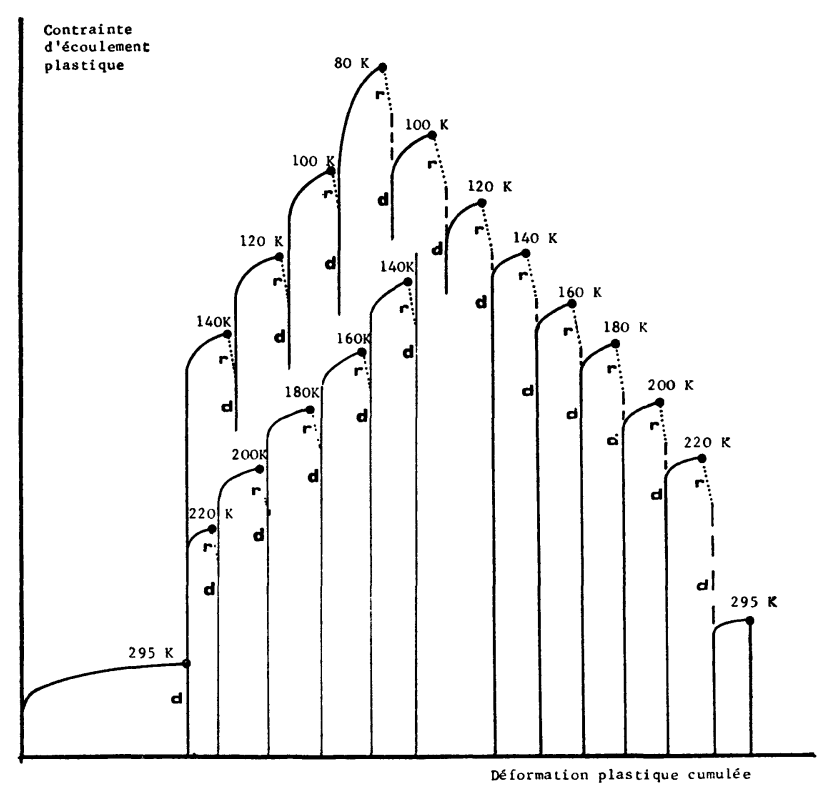

Fig. 5. - Schéma des essais de traction. - éprouvette 4 : programme complet; —— éprouvette 2 : début de programme ; $d$ : décharge et changement de température ; $r$ : relaxation de la contrainte; $\bullet:$ points reportés sur la figure 6 .

[Tensile tests diagram. - sample 4 : complete programme; - sample 2 : partial programme; $d$ : unloading and temperature change ; $r$ : stress relaxation test; $\bullet$ : values plotted on figure 6.]

mée de $2 \%$ à $295 \mathrm{~K}$ pour créer, dans chaque échantillon, des densités comparables de dislocations fraîches. La vitesse de traction est, dans tous les cas, de $0,7 \times 10^{-4} \mathrm{~s}^{-1}$.

A l'issue de la prédéformation, l'éprouvette $n^{0} 4$, par exemple, est déchargée, portée à une température plus basse, $140 \mathrm{~K}$, déformée de $0,5 \%$ par traction 
et la traverse mobile est arrêtée. L'éprouvette relaxe pendant 5 minutes. Si la relaxation était trop faible (volume d'activation trop élevé) une nouvelle déformation de $0,5 \%$, suivie d'une relaxation pourrait être effectuée avant la décharge de l'éprouvette.

Nous avons ainsi obtenu la première partie de la courbe de traction à $140 \mathrm{~K}$ et nous pouvons déterminer la C. E. P. pour $\varepsilon \leqslant 0,5 \%$ ainsi que le volume d'activation associé à là contrainte $\sigma_{0,5}$.

L'éprouvette $\mathrm{n}^{\mathrm{0}} 4$ est alors portée à $120 \mathrm{~K}$, déformée de $0,5 \%$, soumise à un essai de relaxation et déchargée. Nous faisons alors l'hypothèse selon laquelle la C. E. P. déduite de cet essai, après la déformation

$$
\varepsilon_{\mathrm{C}}=0,5 \%(140 \mathrm{~K})+\varepsilon_{\text {relaxation }}+0,5 \%(120 \mathrm{~K})
$$

est la même que la C. E. P. qui aurait été déterminée au cours d'une traction continue effectuée à la température constante de $120 \mathrm{~K}$ pour une déformation égale à $\varepsilon_{\mathrm{C}}$.

L'éprouvette $\mathrm{n}^{0} 4$ est ensuite portée à $100 \mathrm{~K}$, soumise à de nouveaux essais de traction et de relaxation, déchargée, conformément au programme représenté sur la figure 5 .

4.2.2 Application au fer pur (Fig. 6). - Avant d'appliquer cette méthode aux alliages de fer, nous avons voulu vérifier sa validité dans le cas d'un fer pur dont la taille de grains est de $150 \mu \mathrm{m}$ environ.

Les éprouvettes $\mathrm{n}^{\mathrm{os}} 2,3$ et 4 sont déformées conformément au programme précédent, la première traction ayant lieu à $220 \mathrm{~K}, 180 \mathrm{~K}$ et $140 \mathrm{~K}$, respectivement. Les C. E. P. mesurées pour différentes valeurs de $\varepsilon_{C}$, à une même température, nous fournissent une courbe de traction.

Quatre autres éprouvettes, identiques aux précédentes, sont déformées de façon continue à $80 \mathrm{~K}$, $140 \mathrm{~K}, 220 \mathrm{~K}$ et $295 \mathrm{~K}$. Nous constatons que les courbes de traction obtenues par les deux méthodes, sont très voisines les unes des autres, aux basses températures. L'accord est moins bon, à $295 \mathrm{~K}$, pour les fortes déformations.

4.3 Discussion DE LA MÉTHODE. - Le cumul des déformations, tel qu'il est présenté au paragraphe précédent, est acceptable si la C. E. P., à $\varepsilon_{\mathrm{C}}$ et à $T$ données, est indépendante des températures auxquelles les déformations représentées par $\varepsilon_{\mathrm{C}}$ ont été effectuées. La comparaison des courbes obtenues par les deux méthodes montre que si cette hypothèse est vérifiée à basse température, elle ne l'est plus aux températures relativement élevées (courbes à $295 \mathrm{~K}$ : Fig. 6).

En l'absence d'expériences plus détaillées sur ce point, on peut supposer que l'écart observé à $295 \mathrm{~K}$ est explicable de deux façons au moins :

1) Lors des tractions à $200 \mathrm{~K}$ et $220 \mathrm{~K}$ qui précèdent le dernier essai à $295 \mathrm{~K}$, on observe un taux de consolidation $\mathrm{d} \sigma / \mathrm{d} \varepsilon$ très faible. L'initiation d'une légère striction est donc possible, laquelle correspondrait

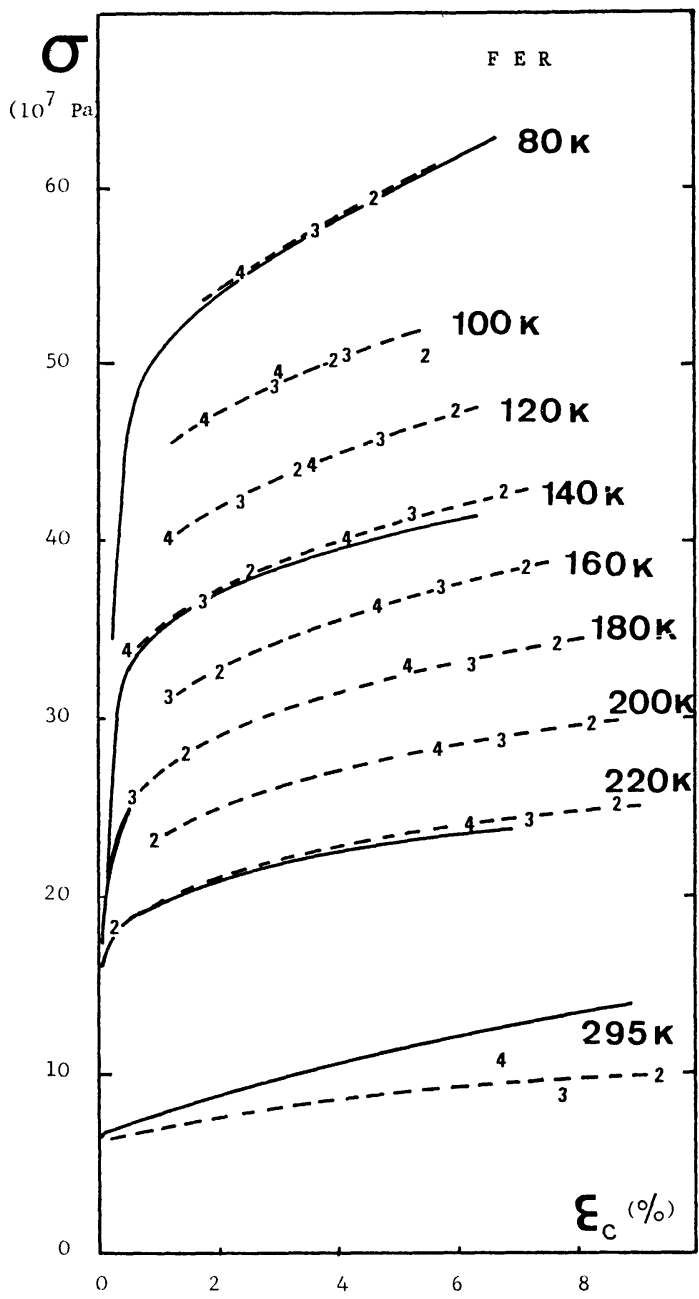

Fig. 6. - Courbes de traction : constante d'écoulement plastique (C. E. P.) en fonction de la déformation plastique cumulée. - en trait continu, courbes de traction isotherme ; - . - - en trait discontinu, courbes de traction déduites des mesures de la C. E. P. à différentes températures sur les éprouvettes 2, 3, 4. [Stress-strain curves: Flow stress versus total plastic strain. — solid line : isothermal tests ; - - - - dotted line : stress-strain curves deduced from flow stress measurements performed on samples 2, 3, 4.]

à une surestimation de la section lors du calcul de la C. E. P.

2) Le passage de $220 \mathrm{~K}$ à $295 \mathrm{~K}$ est relativement long, de l'ordre de l'heure et permettrait à une restauration d'intervenir plus efficacement pendant cette élévation de température que lors d'un essai continu, à $295 \mathrm{~K}$.

En conclusion, les tractions successives, effectuées sur trois éprouvettes seulement, nous permettent de déterminer la C. E.P. d'un alliage fer-X donné, en fonction de la déformation, pour huit températures d'essai, inférieures ou égales à $220 \mathrm{~K}$. De plus, la comparaison des résultats obtenus à partir de plusieurs éprouvettes, à une même température, permet de s'assurer de la reproductibilité des mesures.

Cette méthode est donc bien adaptée à une étude 
systématique de l'adoucissement. Elle reste cependant insuffisante pour la détermination des tout premiers stades de la déformation, indispensable à l'étude physique de ce phénomène.

5. Détermination des paramètres d'activation. - La variation des paramètres d'activation, associée aux phénomènes d'adoucissement, rend leur détermination particulièrement utile à la compréhension des mécanismes responsables de ces effets. Nous avons donc profité des expériences précédentes pour éprouver nos techniques de détermination des paramètres d'activation et contrôler la cohérence de nos mesures avec celles des autres auteurs. Cependant, le nombre des résultats que nous avons obtenus est encore trop faible pour être utilisés lors d'une étude fine des mécanismes de déformation.

5.1 MéTHOde D'Évaluation. - Le volume d'activation est déduit de l'enregistrement de la déformation de l'échantillon, au cours d'un essai de relaxation de la contrainte, cette méthode nous paraissant la plus précise (cf. $\S 3.3 .2$ ).

Nous avons alors adapté les analyses de Guiu et Pratt [40] et de Groh et Conte [41] à la mesure de l'allongement total de l'éprouvette pendant la relaxation.

A partir de la relation :

$-\Delta \tau_{\text {tot }}=\frac{k T}{V} \frac{1}{1+M \theta} \log \left(\frac{1+M \theta}{M} \frac{V \dot{\gamma}_{0}}{k T} t+1\right)$

obtenue avec les mêmes notations par Groh et Conte [41], nous avons admis qu'avec les polycristaux que nous utilisons, $\sigma=2 \tau, \varepsilon=\frac{1}{2} \gamma$.

Dans ces conditions, la déformation plastique de relaxation :

$$
\varepsilon_{\mathrm{p}}=\varepsilon_{\mathrm{total}}-\varepsilon_{\text {elastique }}
$$

est reliée à la contrainte par la relation :

$$
\dot{\varepsilon}_{\mathrm{p}}=-\frac{M}{2} \dot{\tau}_{\text {tot }}
$$

Après report de (3) dans (1), une simple intégration donne l'allongement plastique de relaxation en fonction du temps. Finalement, la prise en compte de (2) permet de relier le volume d'activation apparent à l'allongement total de relaxation que nous mesurons, $\Delta \varepsilon_{\text {tot }}$ :

$V=\frac{2 k T}{\Delta \varepsilon_{\mathrm{tot}}} \frac{S_{0}}{l_{0} d} \frac{1}{1+\left(\frac{S_{0}}{l_{0} d}+\frac{1}{E}\right) \frac{\mathrm{d} \sigma}{\mathrm{d} \varepsilon}} \log \left(\frac{t+c}{c}\right)$

où

$$
c=\frac{1}{\frac{1+M \theta}{M} \frac{V \dot{\gamma}_{0}}{k T}}
$$

peut être considérée comme constante pendant l'essai de relaxation.
L'enthalpie d'activation apparente, $\Delta H$, est alors évaluée à l'aide de la relation classique :

$$
\Delta H=-V T\left(\frac{\partial \tau^{*}}{\partial T}\right)_{j}
$$

qui se transforme, avec nos hypothèses en :

$$
\Delta H=-\frac{V T}{2}\left(\frac{\partial \sigma_{\text {tot }}}{\partial T}\right)_{\dot{\varepsilon}} .
$$

5.2 EXEMPLe DE RÉSUltats. - Les valeurs du volume d'activation en fonction de la C. E. P. à l'instant $t=0$ de la relaxation, sont reportées sur la figure 7 .

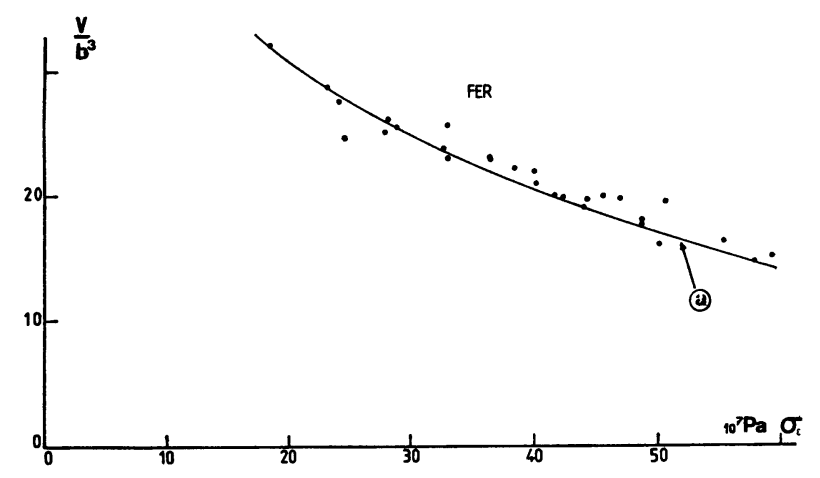

FIG. 7. - Volume d'activation en fonction de la contrainte d'écoulement plastique.

[Activation volume versus flow stress.]

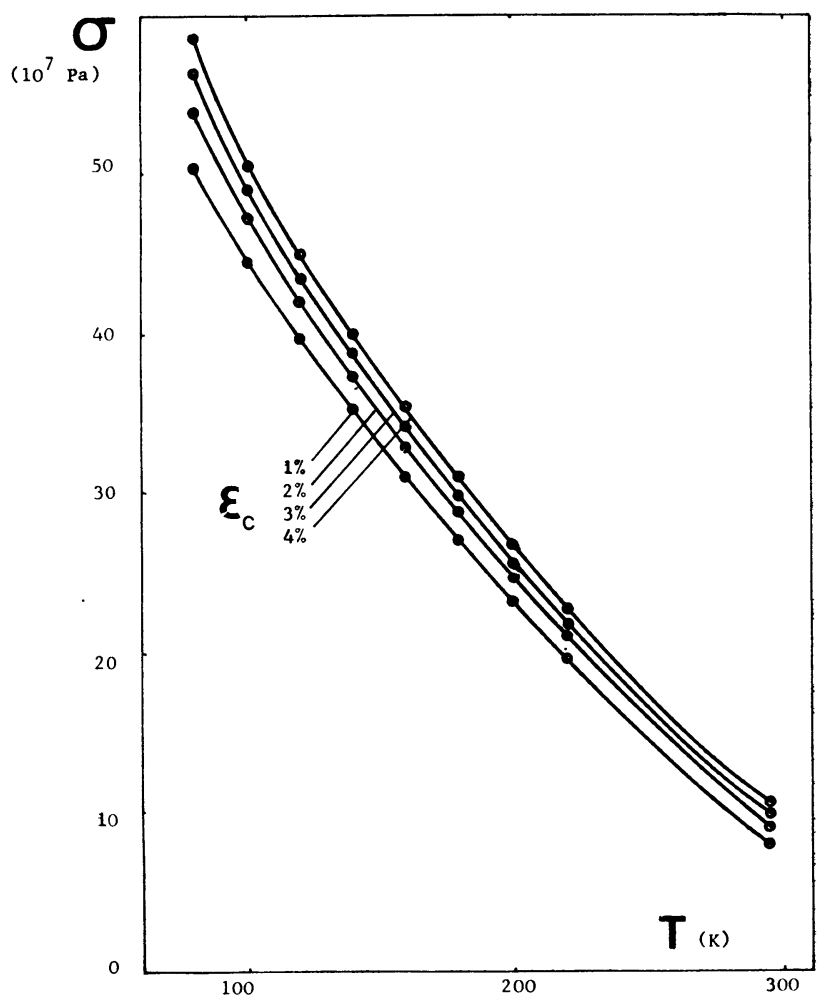

Fig. 8. - Contrainte d'écoulement plastique (C. E. P.) en fonction de la température et de la déformation plastique cumulée $\varepsilon_{\mathrm{C}}$.

[Flow stress versus temperature and total plastic strain $\varepsilon_{\mathrm{c} .]}$ 
L.es points correspondants s'inscrivent bien autour de la courbe continue que nous avions obtenue par mesure de la contrainte en cours de relaxation [3], et nous retrouvons l'ordre de grandeur correct du volume d'activation de la déformation plastique de métaux cubiques centrés à basse température.

L'enthalpie d'activation apparente est calculée à l'aide de la relation (5). Les grandeurs $V$ et $(\partial \sigma / \partial T)_{\varepsilon}$ qui entrent dans ce calcul sont celles qui correspondent à $\sigma_{4} \%$ mesurée à la température $T$ sur les courbes des figures 7 et 8 . Le choix d'une valeur aussi élevée de l'allongement pour déterminer $V$ et $(\partial \sigma / \partial T)$ nous a

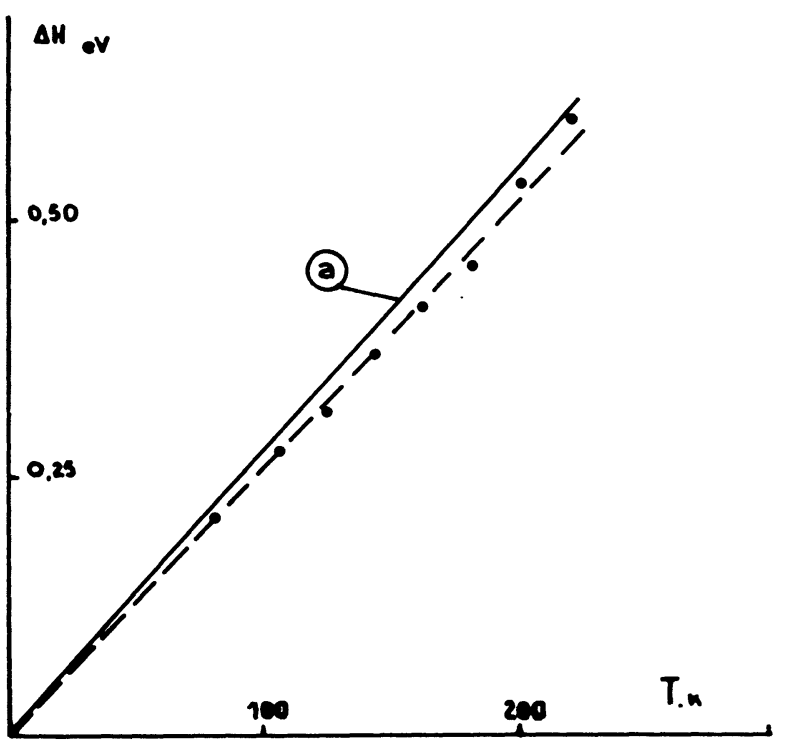

FIG. 9. - Enthalpie d'activation apparente en fonction de la température.

[Apparent activation enthalpy versus temperature.] été dicté par la constatation d'une très faible variation de $\Delta H$ avec la déformation au-delà de $\varepsilon \sim 2 \%$ [39].

Nous pensons alors que l'enthalpie apparente ainsi calculée est bien une grandeur associée au processus de déformation plastique de l'échantillon polycristallin. Les résultats reportés sur la figure 9 montrent que les points expérimentaux se placent bien sur une droite d'équation $\Delta H=C k T$ [42] avec $C=30$. Là encore, nos mesures sont cohérentes avec celles que nous avions effectuées auparavant [3], les énergies mesurées étant du même ordre de grandeur que celles qui ont été publiées par d'autres auteurs [43], [44], [45].

En résumé, les différentes techniques mises au point nous permettent :

- De disposer d'un métal de base de haute pureté ;

- D'allier ce métal à de faibles quantités, bien contrôlées, d'éléments d'alliage se plaçant en substitution, ou en insertion, dans de très bonnes conditions de propreté. Nous pouvons ainsi étudier l'influence d'un seul élément, ou celle de deux éléments associés, sur la C. E. P. du fer, à basse température ;

- De préparer des échantillons dont la taille de grain soit faible, homogène, et identique d'un alliage à l'autre ;

- D'effectuer des essais de traction, et de relaxation mécanique, conduisant à des résultats précis et reproductibles malgré l'emploi d'un petit nombre d'échantillons.

C'est donc en nous appuyant sur ces techniques que nous poursuivons l'étude, déjà commencée au laboratoire [3], [4], [39], du phénomène d'adoucissement du fer, par alliage, à basse température.

\section{Bibliographie}

[1] LesLie, W. C., Metal. Trans. 3 (1972) 5.

[2] Meneses, P., Edelin, G., ICSMA 4, Nancy, p. 296 (1976).

[3] Blanchart, P., Bernadou, J. P., Peyrade, J. P., ChoMEL, P., COTTU, J. P., Script. Met. 9 (1975) 993.

[4] Chomel, P., Cottu, J. P., Peyrade, J. P. (à paraître).

[5] Chaudron, G., Conf. Centre de Perfectionnement Technique, Paris (1967), publiée dans les Ann. Mines (1968) 15.

[6] Duranseaud, J. M., Gayte, F., Odin, G. et Goux, C., C. R. Hebd. Séan. Acad. Sci. C 268 (1969) 469.

[7] Jolly, P., Thèse, Paris (1969).

[8] Jolly, P. et Goux, C., C. R. Hebd. Séan Acad. Sci. C (1969) 1670.

[9] Jolly, P. et Goux, C., Mem. Sci. Rev. Met. 66 (1969) 605.

[10] Talbot, J., Albert, Ph., Caron, M. et Chaudron, G., Rev. Met. 50 (1953) 817.

[11] Talbot, J., Thèse Paris (1955) et Publ. IRSID, série A, $n^{\circ} 137$ (1956)

[12] Vu Quang, K., C. R. Hebd. Séan. Acad. Sci. 260 (1965) 6907,

[13] Vu Quang, K., C. R. Hebd. Séan. Acad. Sci. 262C (1966) 201.

[14] PfanN, W. G., Zone Melting (Wiley, N. Y.) 1958.

[15] Talbot, J., Albert, Ph. et Chaudron, G., C. R. Hebd. Séan. Acad. Sci. 244 (1957) 1577.
[16] Rutherford, J. L., Smith, R. L., Herman, M. et SpanGLER, G. E., Coll. Int. C. N. R. S., Paris, p. 201 (1959).

[17] Smith, R. L. et Koepel, E. J., Coll. Int. C. N. R. S., Paris, p. 207 (1966).

[18] Galtier, F., Prince-Ringuet, F. Le, Reboux, J., Collongues, P. et Chaudron, G., C. R. Hebd. Séan. Acad. Sci. 255 (1962) 2539.

[19] Rondot, B., Antoniucci, P., Montuelle, J. et ChauDRON, G., C. R. Hebd. Séan. Acad. Sci. 266C (1968) 363.

[20] Jenkins, A. E., Harris, B. and Baker, L., Symposium of Metallurgy at high pressures and temperatures, Met. Soc. AIME, Conf. (Gordon and Breach, N. Y.) 1964, vol. 22.

[21] Peifer, W. A., J. Met. 17 (1965) 487.

[22] Rony, P., Trans. Vac. Met. Conf., Am. Vac. Soc. Boston, Mass. (1964) p. 55.

[23] Bunshah, R. F., Tech. Met. Res. I, part. 2, Chap. 18.

[24] Smith, R. L. and Koepel, E. J., Mem. Scient. de la Rev. de métallurgie, Colloque sur le fer de très haute pureté (1968).

[25] Chaudron, G., Monographies sur les métaux de haute pureté : Fer (1972).

[26] Richardson, M. and JefFes, J. H. E., J. Iron Steel Inst. 160 (1948) 261. 
[27] Oliver, B. F., Troy, E. W., Oxydation zone refining, Colloque sur le fer de très haute pureté (1968).

[28] KUBACHEWSKI, M., EvANS, E., La thermochimie en métallurgie (Gauthier Villars) 1957.

[29] Bodsworth, C., Appeton, A. S., Problems in applied thermodynamics par Longmans (Green and Co L. T. D. Londres).

[30] Wyjadlowski, T., Rochette, J., TaRdy, R. et Boos, J. Y., Lettre à l'éditeur, Mem. Sci. Rev. Met., janvier (1973).

[31] Dimitrov, O., Centre d'Etudes de Chimie Métallurgique, Colloque sur le fer de très haute pureté (1968).

[32] VANONI, F., Communication privée.

[33] Peyrade, J. P., Astie, P., Garigue, J., Mem. Sci. Rev. Mét. LXXI (1974).

[34] Peyrade, J. P., Garigue, J., Degauque, J., C. R. Hebd. Séan. Acad. Sci. 273C (1971) 1500-1053.
[35] Peyrade, J. P., Thèse $3^{e}$ Cycle, Toulouse (1971).

[36] Vanoni, F., Thèse de Doctorat d'Université, Grenoble (1973).

[37] Antonione, C., Della-Gatta, Lucci, A., Riontino, G., Venturello, G., Acta Met. 18 (1970) 1169.

[38] Askenazy, S., Carrere, G., Marquez, J., Colloques Internationaux du C. N. R. S., $\mathrm{n}^{\circ}$ 242, Grenoble (1974).

[39] Blanchart, P., Thèse de $3^{\circ}$ Cycle, Toulouse (1975).

[40] Guiv, F., Pratt, P. L., Phys. Stat. Solids 6 (1964) 111.

[41] Groh, P., Conte, R., Acta Met. 19 (1971) 895.

[42] KuBIN, L. P., Thèse de Doctorat, Orsay (1971).

[43] Conte, R., Groh, P., Escaig, B., Phys. Stat. Solids 28 (1968) 475.

[44] NaKadA, Y., KeH, A. S., Acta Met. 16 (1968) 903.

[45] Kubin, L. P., LOUChet, F., Groh, P., VANONI, F., ICSMA 4, p. 814 , Nancy (1976). 